This document is confidential and is proprietary to the American Chemical Society and its authors. Do not copy or disclose without written permission. If you have received this item in error, notify the sender and delete all copies.

\title{
The Endofullerene HF@C60: Inelastic Neutron Scattering Spectra from Quantum Simulations and Experiment, Validity of the Selection Rule and Symmetry Breaking
}

\begin{tabular}{|r|l|}
\hline Journal: & The Journal of Physical Chemistry Letters \\
\hline Manuscript ID & jz-2019-02005s \\
\hline Manuscript Type: & Letter \\
\hline Author: & $10-J u l-2019$ \\
\hline Complete List of Authors: & $\begin{array}{l}\text { Xu, Minzhong; New York University, Department of Chemistry } \\
\text { Felker, Peter; University of California Los Angeles, Department of } \\
\text { Chemistry and Biochemistry } \\
\text { Mamone, Salvatore; University of Nottingham, School of Physics and } \\
\text { Astronomy } \\
\text { Horsewill, Anthony; University of Nottingham, School of Physics and } \\
\text { Astronomy } \\
\text { Rols, Stephane; ILL, } \\
\text { Whitby, Richard; University of Southampton, Chemistry } \\
\text { Bacic, Zlatko; New York University, Department of Chemistry }\end{array}$ \\
\hline
\end{tabular}

\section{SCHOLARONE \\ Manuscripts}




\title{
The Endofullerene $\mathrm{HFOC}_{60}$ : Inelastic Neutron Scattering Spectra from Quantum
}

\section{Simulations and Experiment, Validity of the Selection Rule and Symmetry Breaking}

\author{
Minzhong $\mathrm{Xu},{ }^{*} \dagger$ Peter M. Felker, ${ }^{\ddagger}$ Salvatore Mamone, Anthony J. Horsewill,

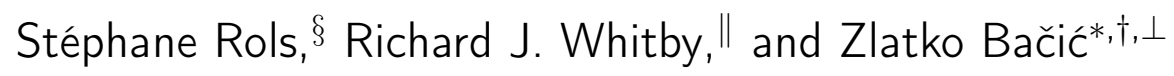 \\ $\dagger$ †epartment of Chemistry, New York University, New York, New York 10003, United \\ States \\ $\ddagger$ Department of Chemistry and Biochemistry, University of California, Los Angeles, \\ California 90095-1569, United States \\ 9Shool of Physics $\& 3$ Astronomy, University of Nottingham, Nottingham NG7 2RD, United \\ Kingdom \\ §Institut Laue-Langevin, CS 20156, 38042 Grenoble, France \\ \|Chemistry, Faculty of Engineering and Physical Sciences, University of Southampton, \\ Southampton, SO17 1BJ, United Kingdom \\ $\perp N Y U$-ECNU Center for Computational Chemistry at NYU Shanghai, 3663 Zhongshan \\ Road North, Shanghai 200062, China \\ E-mail: $m \times 200 @ n y u . e d u ; ~ z l a t k o . b a c i c @ n y u . e d u$
}




\begin{abstract}
Accurate quantum simulations of the low-temperature inelastic neutron scattering (INS) spectra of $\mathrm{HF}_{\mathrm{C}} \mathrm{C}_{60}$ are reported, for two incident neutron wavelengths. They are distinguished by the rigorous inclusion of symmetry-breaking effects in the treatment, and having the spectra computed with $\mathrm{HF}$ as the guest, rather than $\mathrm{H}_{2}$ or $\mathrm{HD}$, as in the past work. The results demonstrate that the precedent-setting INS selection rule, originally derived for $\mathrm{H}_{2}$ and $\mathrm{HD}$ in near-spherical nanocavities, applies also to $\mathrm{HF} @ \mathrm{C}_{60}$, despite the large mass asymmetry of $\mathrm{HF}$ and the strongly mixed character of its translation-rotation eigenstates. This lends crucial support to the theoretical prediction [ Xu, M.; et al. J. Phys. Chem. Lett. 2015, 6, 3721] that the INS selection rule is valid for any diatomic molecule in near-spherical nanoconfinement. The selection rule remains valid in the presence of symmetry breaking, but is modified slightly in an interesting way. Comparison is made with the recently published experimental INS spectrum of HF@ $\mathrm{C}_{60}$. The agreement is very good, apart from one peak for which our calculations suggest a reassignment. This reassignment is consistent with the measured INS spectrum presented in this work, that covers an extended energy range.
\end{abstract}

\title{
Graphical TOC Entry
}

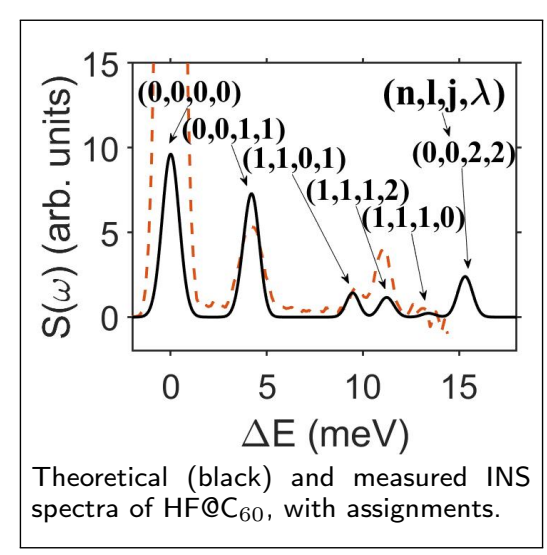


Light-molecule endofullerenes have molecules such as $\mathrm{H}_{2}, \mathrm{H}_{2} \mathrm{O}, \mathrm{HF}$, and $\mathrm{CH}_{4}$, characterized by small masses and large rotational constants, encapsulated inside the cages of $\mathrm{C}_{60}, \mathrm{C}_{70}$ and other fullerenes. ${ }^{1,2}$ Their synthesis has been accomplished utilizing the approach known as molecular surgery, in which a hole is created in the fullerene cage through a sequence of chemical reactions allowing the insertion of the guest molecule into the cavity, followed by reforming the pristine fullerene shell with the guest molecule trapped in its interior. ${ }^{3-6}$ Inside $\mathrm{C}_{60}$, the entrapped molecules are under the conditions unlike any other studied previously confined in the highly symmetric and nonpolar nanoscale cage and, in the case of HF and $\mathrm{H}_{2} \mathrm{O}$, isolated and unable to form hydrogen bonds to other molecules.

Another distinguishing feature of light-molecule endofullerenes, one that has motivated a large number of experimental and theoretical studies, is the dominance of quantum effects in the dynamics and spectroscopy of the guest molecules. ${ }^{1,2,7}$ These species exemplify many of the fundamental, textbook principles of quantum mechanics to the degree and with the clarity that are unmatched among molecular systems amenable to experimental investigations. One source of quantum effects is the quantization of the translational center-of-mass (c.m.) degrees of freedom (DOFs) of the encapsulated molecules (particle-in-a-box effect). Tight confinement gives rise to translational eigenstates whose energy differences are large relative to $k T$ ( $k$ being the Boltzmann constant). The same holds for the quantized rotational states of the light molecules. The quantized translational and rotational DOFs of the guest molecule are coupled by the confining potential of the fullerene interior, resulting in a sparse and intricate translation-rotation (TR) energy level structure. ${ }^{1,7}$

The endofullerene $\mathrm{HF} @ \mathrm{C}_{60}$, where the fullerene cage is occupied by a single dipolar $\mathrm{HF}$ molecule, is of considerable interest to experimentalists and theorists alike. Among its attractions is the opportunity that electric-dipole-coupled arrays of close-packed $\mathrm{HF} @ \mathrm{C}_{60}$ moieties provide for investigating the many-body correlations between the highly quantum molecular dipoles confined in the $\mathrm{C}_{60}$ cages, and the possible emergence of dipole-ordered phases and ferroelectricity at low temperatures. ${ }^{8}$ The inelastic neutron scattering (INS) 
and far- and mid-infrared (IR) spectra have revealed quantization of the translational and rotational DOFs of the encapsulated HF. ${ }^{5}$ Moreover, both the rotational transitions and the intramolecular stretch fundamental of the caged HF are shifted to lower energies relative to those in the gas phase, evidence for the softening of the HF intramolecular potential caused by the guest-host interaction. ${ }^{5}$ Finally, in the far- and mid-IR spectra of solid HF@ $\mathrm{C}_{60}$ at low temperature $(5 \mathrm{~K}),{ }^{5}$ the rotational $j=0 \rightarrow 1$ transition is split by $3.9 \mathrm{~cm}^{-1}$, signaling that the symmetry of the environment felt by the guest HF must be lower than $I_{h}$, the symmetry of an isolated $\mathrm{C}_{60}$ molecule. Symmetry breaking was previously observed in solid $\mathrm{H}_{2} @ \mathrm{C}_{60} 9,10$ and $\mathrm{H}_{2} \mathrm{O} @ \mathrm{C}_{60}{ }^{11-13}$ at low temperatures.

These experimental findings have stimulated a number of theoretical studies of the quantum TR dynamics and spectroscopy of $\mathrm{HF} @ \mathrm{C}_{60}$. Quantum-five dimensional (5D) boundstate calculations for the isolated $\mathrm{HF} @ \mathrm{C}_{60}$ (treating both $\mathrm{HF}$ and $\mathrm{C}_{60}$ as rigid) ${ }^{14,15}$ employing the recent $a b$ initio 5D PES of the system ${ }^{14}$ have revealed that, despite the large mass asymmetry of HF, its TR eigenstates exhibit many of the salient features of the TR level structure of $\mathrm{H}_{2} @ \mathrm{C}_{60}$ (apart from spin isomerism), elucidated by theory previously. ${ }^{7,16-19}$ These include the use of $(i)$ the principal and orbital angular momentum quantum numbers $n$ and $l$, respectively, of the 3D isotropic harmonic oscillator (HO) for the translational c.m. excitations of $\mathrm{HF}$, $(i i)$ the quantum number $j$ of a linear rigid rotor for the HF rotational excitations, (iii) and the total angular momentum quantum number $\lambda$, arising from the vectorial coupling of the orbital angular momentum $\boldsymbol{l}$ associated with the translational motion of HF and the rotational angular momentum $\boldsymbol{j}$ of the molecule. In addition, the recently developed first-principles electrostatic quadrupolar model ${ }^{20}$ has been shown to account quantitatively for the effects of symmetry breaking on both the $j=1$ level of $\mathrm{HF}^{20}$ and its excited TR eigenstates $^{15}$ (as well as the corresponding TR levels of the caged $\mathrm{H}_{2}$ and $\mathrm{H}_{2} \mathrm{O}$ ).

Here we present rigorous quantum simulations of the low-temperature INS spectra of $\mathrm{HF} @ \mathrm{C}_{60}$ for two incident neutron wavelengths, utilizing the methodology developed by Bačić, $\mathrm{Xu}$, and co-workers. ${ }^{1,21-23}$ These calculations are notable for several reasons. For the first 
time, they include explicitly the effects of symmetry breaking on the quantum 5D TR eigenstates of the encaged molecule, HF, and hence the INS spectra, since the former constitute the initial and final states of the INS transitions. These simulations are also the first to involve a guest molecule other than $\mathrm{H}_{2}$ or HD. Therefore, they make it possible to establish whether the first ever (and so far the only) selection rule established in the INS spectroscopy of discrete molecular compounds, ${ }^{24}$ and validated experimentally for $\mathrm{H}_{2} @ \mathrm{C}_{60},{ }^{25}$ applies to $\mathrm{HF} @ \mathrm{C}_{60}$ as well, as suggested by the generalized INS selection rule. ${ }^{26}$ If it does, is it modified in some way by the symmetry breaking? Finally, comparison is made between the INS spectra computed in the present study and the experimental spectrum in the literature, ${ }^{5}$ suggesting the reassignment of one peak in the latter. This reassignment is consistent with the measured INS spectrum presented in this work, that covers an extended energy range.

The quantum methodology employed in this study to calculate accurately the INS spectra of $\mathrm{HF} @ \mathrm{C}_{60}$, with and without the inclusion of symmetry breaking, is outlined in section $\mathrm{S} 1$ of the Supporting Information, and presented in full in ref 23. The TR levels and wave functions of $\mathrm{HF} @ \mathrm{C}_{60}$, that serve as input for the simulations of the INS spectra, were calculated in ref 15, using the approach summarized in section S2 of the Supporting Information.

Table 1 shows the intensities of the INS transitions out of the ground TR state of $\mathrm{HF} @ \mathrm{C}_{60}$ to its excited TR levels, for the incident neutron wavelength $\lambda_{\mathrm{n}}=1.65 \AA$ (one of the two wavelengths for which the INS spectra were recorded), calculated in this work for the isolated endofullerene $\left(I_{0}\right)$ and for the dominant $\mathrm{P}$ crystal orientation $\left(I_{P}\right)$ of $\mathrm{HF} @ \mathrm{C}_{60}(s)$, respectively. The $\mathrm{P}$ and $\mathrm{H}$ crystal orientations are defined in section S1 of the Supporting Information. For the $\mathrm{H}$ orientation, the calculated symmetry-induced splittings of the TR levels are about a factor of 30 smaller than the corresponding ones in the $\mathrm{P}$ orientation. ${ }^{20}$ Also displayed in Table 1 are select lower-lying TR levels of $\mathrm{HF}_{\mathrm{C}} \mathrm{C}_{60}$, the final states of the INS transitions considered, computed for the $\mathrm{P}$ crystal orientation. For each level, we show its energy $\left(\Delta E_{P}\right)$ relative to the TR ground state, its degeneracy $\left(g_{P}\right)$, and the quantum numbers $(n, l, j), \lambda$, and $\left|m_{\lambda}\right|$. Those levels whose parentage derives from the same level of the isolated HF@C 60 
are grouped together. The energies $\left(\Delta E_{0}\right)$ and degeneracies $\left(g_{0}\right)$ of these "parent" levels are given in the first two columns of Table 1. Both sets of TR levels are from ref 15.

The information presented in Table 1 allows us to ascertain if the unexpected INS selection rule that we established ${ }^{24}$ and subsequently generalized, ${ }^{26}$ is valid for $\mathrm{HF} @ \mathrm{C}_{60}$. The discovery of this selection rule was very surprising, since it contradicted the view widely held by the INS community that the INS spectroscopy of discrete molecular compounds is not subject to any selection rules. ${ }^{27-30}$

The selection rule in question, in its original form ${ }^{24}$ states that for a para- $\mathrm{H}_{2}$ or HD molecule confined in a near-spherical nanocavity, such as that of $\mathrm{C}_{60}$, the INS transitions between its ground TR state and excited TR states assigned as $(n, l, j, \lambda)$ are forbidden for $\lambda=j+l-1$. A later derivation showed that this selection rule, and its generalized version, should apply to any diatomic molecule, homo- or heteronuclear, inside a near-spherical nanocavity. ${ }^{26}$ These derivations ${ }^{24,26}$ involve explicit evaluation of the INS transition matrix element, and a physically motivated assumption about the functional form of the radial component of the TR eigenstates. $\mathrm{HF} @ \mathrm{C}_{60}$ poses an important test of the generality of the selection rule, and a demanding one, given the large mass asymmetry of the molecule, and the fact that, as elaborated below, $n, l, j$ are not nearly as good quantum numbers for HF as they are for $\mathrm{H}_{2} \cdot{ }^{15}$ It should be pointed out that the group-theoretical derivation of these selection rules ${ }^{31}$ is strictly applicable only to homonuclear diatomic molecules such as $\mathrm{H}_{2}$ inside a spherical cavity (leaving out even $I_{h}$ systems like $\mathrm{C}_{60}$ ), and says nothing about caged heteronuclear diatomics like $\mathrm{HF}$ (or $\mathrm{HD}^{32}$ ).

In Table 1, the bold-faced transitions are those that for isolated $\mathrm{HF} @ \mathrm{C}_{60}$ should be forbidden according to the above selection rule. Indeed, their intensities $\left(I_{0}\right)$ are zero to the number of significant figures shown, implying that these transitions are unobservable in the INS spectra, and thus forbidden for all practical purposes. This provides a computational confirmation of the validity of the INS selection rule for this system, as derived by us. ${ }^{24,26}$ It also attests that the scope of validity of the selection rule is significantly larger than 
suggested by the group-theoretical considerations. ${ }^{31}$

Table 1 also shows how the INS selection rule is affected by the symmetry breaking. It is clear that symmetry breaking splits the TR levels of isolated $\mathrm{HF} @ \mathrm{C}_{60}$ with the quantum number $\lambda$, in the $\Delta E_{0}$ column, into $\lambda+1$ sublevels shown in the $\Delta E_{P}$ column, each associated with one value of $\left|m_{\lambda}\right|$. The same of course happens to the INS transitions to these TR levels. Looking at each of the transitions that for isolated $\mathrm{HF} @ \mathrm{C}_{60}$ is forbidden, one sees that in the presence of symmetry breaking only the transition to the $\left|m_{\lambda}\right|=0$ sublevel remains forbidden, with the intensity that is zero to four significant figures. But, the intensities of the transitions to the $\left|m_{\lambda}\right|>0$ sublevels, while nonzero, are very small, and would be invisible in the INS spectrum as well. The relationship between these predictions and the available experimental spectra is discussed later in the paper.

One important point warrants discussion here. The INS selection rule ${ }^{24,26}$ is expressed in terms of the TR quantum numbers $n, l, j$ and $\lambda$, that are assumed to be good quantum numbers. This is certainly the case for $\mathrm{H}_{2}{ }^{15,17}$ (and $\mathrm{H}_{2} \mathrm{O}^{15}$ ) in $\mathrm{C}_{60}$. However, as mentioned above, that is not true for $\mathrm{HF} @ \mathrm{C}_{60}$, apart from the quantum number $\lambda .{ }^{15}$ Let us consider two TR eigenstates in Table 1 that are the respective final states of two forbidden INS transitions. One of them, $(2,2,1,2)$ at $21.222 \mathrm{meV}\left(\left|m_{\lambda}\right|=0\right)$, is $52.1 \%|2,2,1\rangle+45.2 \%|1,1,2\rangle$. Given such strong mixing of two sets of $(n, l, j)$ quantum numbers, why does the INS selection rule apply to this TR eigenstate? The reason is that both $|2,2,1\rangle$ and $|1,1,2\rangle$ separately satisfy the selection rule condition $\lambda=j+l-1$, with $\lambda=2$. Likewise, the second "forbidden" TR eigenstate $(1,1,2,2)$, at $24.162 \mathrm{meV}\left(\left|m_{\lambda}\right|=0\right)$, is strongly mixed as well, $52.8 \%|1,1,2\rangle+$ $44.8 \%|2,2,1\rangle$. But the transition to it (from the ground TR state) is forbidden since $|1,1,2\rangle$ and $|2,2,1\rangle$ separately satisfy the condition $\lambda=j+l-1$, for $\lambda=2$ [the lowest-energy "forbidden" eigenstate $(1,1,1,1)$, at $13.683 \mathrm{meV}$ in Table 1 , happens to be a remarkably pure state, $97 \%|1,1,1\rangle]$.

Precisely the same was established earlier for $\mathrm{HD} @ \mathrm{C}_{60},{ }^{26}$ another system whose TR eigenstates have strongly mixed character. ${ }^{17}$ Thus, we can now make a general statement 
about the reason why the INS selection rule applies to heteronuclear, mass-asymmetric systems such as $\mathrm{HF} @ \mathrm{C}_{60}$ and $\mathrm{HD} @ \mathrm{C}_{60}$, whose TR eigenstates are strongly mixed and $(n, l, j)$ are not particularly good quantum numbers. The reason is that each basis state having a significant weight in a "forbidden" TR eigenstate individually satisfies the selection rule condition $\lambda=j+l-1$.

Figure 1 shows the INS spectrum of $\mathrm{HF} @ \mathrm{C}_{60}$ computed for $0 \mathrm{~K}$ and the incident neutron wavelength $\lambda_{\mathrm{n}}=2.22 \AA$ and, in Figure 1a, the INS spectrum recorded at $1.6 \mathrm{~K}$ using the same incident neutron wavelength. ${ }^{5}$ At these temperatures, the INS transitions, calculated and observed, all originate from the ground TR state of HF, since it is the only one populated. Figures $1 \mathrm{~b}$ and $1 \mathrm{c}$ show the stick spectra computed for the $\mathrm{P}$ and $\mathrm{H}$ crystal orientations, respectively. The theoretical spectrum in Figure $1 \mathrm{a}$ is a weighted sum of the $\mathrm{P}$ and $\mathrm{H}$ stick spectra, computed for a $15 \%$ contribution from the $\mathrm{H}$ orientation and $85 \%$ from the $\mathrm{P}$ orientation (corresponding to the measured abundances of the two crystal types under the experimental conditions). It is then convolved with the instrumental resolution function. The transitions are labeled with the quantum numbers $(n, l, j, \lambda)$ of their final TR states.

The first peak of the calculated INS spectrum in Figure 1a corresponds to a closely spaced pair of rotational transitions to the $\left|m_{\lambda}\right|=0$ and 1 sublevels resulting from the 1:2 splitting of the $j=1$ level $(0,0,1,1)$ of HF (visible in Figure $1 \mathrm{~b}$ ). The splitting of $0.461 \mathrm{meV}$ is not resolved in either the calculated (convolved) or measured INS spectrum. The energies of the two transitions, 3.88 and $4.34 \mathrm{meV}$ (Table 1), agree well with the measured peak centered at $4.1 \mathrm{meV}$, confirming its assignment as the rotational $j=0 \rightarrow 1$ transition. $^{5}$

The next higher-energy peak in the calculated spectrum arises from the transition to the HF translational fundamental $(1,1,0,1)$, whose $\left|m_{\lambda}\right|=0$ and 1 sublevels are split by only $0.02 \mathrm{meV} .{ }^{15}$ The two (unresolved) transitions at 9.45 and $9.47 \mathrm{meV}$, respectively, are in good agreement with the peak in the experimental spectrum at $9.75 \mathrm{meV}$. This peak was tentatively assigned to the fundamental translational transition, ${ }^{5}$ and our calculations support this assignment. 
The peak visible at $11 \mathrm{meV}$ in both the computed and measured spectra was assigned by Krachmalnicoff et al. ${ }^{5}$ to the $j=0 \rightarrow j=2$ rotational transition. However, as can be seen in Table 1, the only calculated transitions near this energy are those to the $\lambda=2$ sublevel of the $n=1, j=1$ multiplet $(1,1,1,2)$ (triplet in $I_{h}$ symmetry), split by the symmetry breaking into three components corresponding to $\left|m_{\lambda}\right|=0,1$, and 2 , respectively, in the energy range 11.06 - $11.45 \mathrm{meV}$. These three transitions are visible in the stick spectrum in Figure $1 \mathrm{~b}$. The transition to the $\lambda=0$ sublevel $(1,1,1,0)$ at $13.41 \mathrm{meV}$ has a low intensity, and appears in the calculated spectrum only as a slight bump. Unfortunately, the measured INS spectrum presented in Figure 3a of ref 5 ends abruptly beyond $11 \mathrm{meV}$, precluding the comparison with the calculated spectrum at higher energies.

If the peak observed, and calculated, around $11 \mathrm{meV}$ arises from transitions to the $n=$ $1, j=1$ multiplet $(1,1,1,2)$, then where is the peak associated with the $j=0 \rightarrow j=2$ rotational transition? According to the calculations, Table 1 and Figure 1a, the transition to the $j=2$ rotational level $(0,0,2,2)$ of $\mathrm{HF}$, that is split into three components with $\left|m_{\lambda}\right|=0,1$, and 2 , respectively (see Figure $1 \mathrm{~b}$ ), appears in the range $15.17-15.50 \mathrm{meV}$. But, as mentioned above, the measured INS spectrum in ref 5 terminates around $11 \mathrm{meV}$.

Fortunately, the INS spectrum of $\mathrm{HF} @ \mathrm{C}_{60}$, recorded at $1.6 \mathrm{~K}$ but with a shorter incident neutron wavelength $\lambda_{\mathrm{n}}=1.60 \AA$ is available, and is shown in Figure 2. The INS measurements were conducted on the IN4C thermal time-of-flight spectrometer located at the Institut Laue Langevin in Grenoble (France). Additional information is available in section $\mathrm{S} 3$ of the Supporting Information. This spectrum, extending beyond $11 \mathrm{meV}$, does exhibit a prominent peak around $16 \mathrm{meV}$, that agrees well with our calculated transition to the $j=2$ rotational level $(0,0,2,2)$. One may be surprised by the rather high energy of this TR level, given that the $j=0 \rightarrow 1$ transition is around $4 \mathrm{meV}$. But as pointed out above, $(n, l, j)$ are not very good quantum numbers for $\mathrm{HF} @ \mathrm{C}_{60}$. Thus, the $\mathrm{TR}$ eigenstate $(0,0,2,2)$ is only $62 \%|0,0,2\rangle$, so its assignment as the $j=2$ level is not to be taken literally.

The broad asymmetric band with the maximum at $11 \mathrm{meV}$ in Figure 2 covers the energy 
range from 8 to $12 \mathrm{meV}$. Hidden underneath are the peaks around 9.75 and $11 \mathrm{meV}$ that are resolved for $\lambda_{\mathrm{n}}=2.22 \AA$ (Figure 1a). The former is assigned, by experiment and theory, to the transitions to the HF translational fundamental, and the latter (by us in this study) to the $n=1, j=1$ multiplet, respectively. The peak around $4 \mathrm{meV}$ matches that recorded for $\lambda_{\mathrm{n}}=2.22 \AA$ (Figure 1a) and has been already assigned to the transitions to the symmetrysplit $j=1$ rotational level of $\mathrm{HF}$.

Figure 3 displays the INS spectrum of $\mathrm{HF}_{6} \mathrm{C}_{60}$ computed for $0 \mathrm{~K}$ and the incident neutron wavelength $\lambda_{\mathrm{n}}=1.65 \AA$, that can be thought of as the theoretical counterpart to the measured INS spectrum in Figure 2. What this simulated spectrum shows is that beyond $16 \mathrm{meV}$ there are no intense transitions, so that in this energy range the measured INS spectrum should have few, if any, distinctive features.

Can the two experimental INS spectra that are currently available be used to validate the predictions of the INS selection rule presented earlier in the paper? The lowest-energy (bold-faced) transition in Table 1 predicted to be forbidden, to the $\left|m_{\lambda}\right|=0$ component of the $n=1, j=1$ doublet $(1,1,1,1)$, lies at $13.68 \mathrm{meV}$. This is beyond the range of the INS spectrum measured in ref 5 , but not of that in Figure 2. The latter spectrum appears to show a dip, or absence of any features, between 13 and $15 \mathrm{meV}$, which is consistent with the selection rule. However, a better resolved and less noisy INS spectrum is needed for a definitive experimental confirmation of the validity of the selection rule for $\mathrm{HF} @ \mathrm{C}_{60}$.

In summary, we have presented rigorous quantum simulations of the low-temperature INS spectra of $\mathrm{HF} @ \mathrm{C}_{60}$, employing the methodology developed several years ago. ${ }^{21-23}$ They are distinctive in a number of ways. The effects of symmetry breaking ${ }^{15,20}$ are included explicitly for the first time in the calculations of the INS spectra. Also for the first time in the INS calculations for light-molecule endofullerenes, ${ }^{1}$ the guest molecule, $\mathrm{HF}$, is not $\mathrm{H}_{2}$ or one of its isotopologues. This has allowed us to test the prediction ${ }^{26}$ that the INS selection rule initially derived for $\mathrm{H}_{2}$ or $\mathrm{HD}$ in a near-spherical nanocavity ${ }^{24,32}$ applies to a much broader class of diatomic molecules in such a confinement. HF presents a challenge, owing 
to its large mass asymmetry and because $n, l, j$ are not nearly as good quantum numbers as they are for $\mathrm{H}_{2} .{ }^{15}$ Nevertheless, the INS selection rule completely passed the (computational) test, both for the isolated $\mathrm{HF} @ \mathrm{C}_{60}$, without symmetry breaking, and when the symmetry breaking is taken into account (modifying the selection rule in a particular way). The measured INS spectrum that encompasses the lowest-energy computed forbidden transition is not inconsistent with this band being forbidden. But, an unambiguous verification of the theory requires an experimental INS spectrum of $\mathrm{HF} @ \mathrm{C}_{60}$ that is better resolved and less noisy. Our calculations agree with the assignments of two out of three peaks visible in the

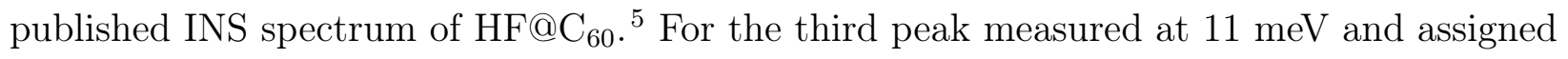
to the $j=0 \rightarrow j=2$ rotational transition, ${ }^{5}$ we suggest its reassignment to the closely spaced $n=1, j=1$ multiplet. The calculations predict that the transitions to the $j=2$ rotational level, split by symmetry breaking, are in the range $15.17-15.50 \mathrm{meV}$. This is in good agreement with the the INS spectrum of $\mathrm{HF} @ \mathrm{C}_{60}$ recorded over an extended energy range, in which a peak around $16 \mathrm{meV}$ figures prominently. Evidently, the close interaction between high-level theory and experiment, that has been the hallmark of this field, continues to be stimulating and productive, leading to deeper understanding of these intriguing systems. In particular, the results of this study support the contention made by us previously, ${ }^{26}$ that the INS selection rule applies to any diatomic molecule in near-spherical confinement. This fundamental topic should be pursued further by experiment and theory.

\section{Acknowledgement}

Z.B. thanks the National Science Foundation for financial support of this research through the Grant CHE-1566085. R.J.W., A.J.H. and S.M. thank the Engineering and Physical Sciences Research Council for funding through grants EP/I029451/1, EP/M001962/1 and $\mathrm{EP} / \mathrm{M} 001970 / 1$. 


\section{Supporting Information Available}

The following is available free of charge.

- Overview of (a) the methodology for quantum 5D calculation of the translation-rotation eigenstates of $\mathrm{HF} @ \mathrm{C}_{60}$ including symmetry breaking, and (b) rigorous quantum treatment of the inelastic neutron scattering spectra of $\mathrm{HF} @ \mathrm{C}_{60}$, as well as a brief description of the INS measurements.

This material is available free of charge via the Internet at http://pubs.acs.org/.

\section{References}

(1) Bačić, Z. Perspective: Accurate treatment of the quantum dynamics of light molecules inside fullerene cages: Translation-rotation states, spectroscopy, and symmetry breaking. J. Chem. Phys. 2018, 149, 100901.

(2) Levitt, M. H. Spectroscopy of light-molecule endofullerenes. Phil. Trans. R. Soc. A 2013, 371, 20120429.

(3) Komatsu, K.; Murata, M.; Murata, Y. Encapsulation of molecular hydrogen in fullerene $\mathrm{C}_{60}$ by organic synthesis. Science 2005, 30\%, 238.

(4) Kurotobi, K.; Murata, Y. A single molecule of water encapsulated in fullerene $\mathrm{C}_{60}$. Science 2011, 333, 613.

(5) Krachmalnicoff, A.; Bounds, R.; Mamone, S.; Alom, S.; Concistrè, M.; Meier, B.; Kouřil, K.; Light, M. E.; Johnson, M. R.; Rols, S. et al. The dipolar endofullerene HF@C 60. Nature Chem. 2016, 8, 953.

(6) Bloodworth, S.; Sitinova, G.; Alom, S.; Vidal, S.; Bacanu, G. R.; Elliott, S. J.; Light, M. E.; Herniman, J. M.; Langley, G. J.; Levitt, M. H. et al. First synthesis and characterization of $\mathrm{CH}_{4} @ \mathrm{C}_{60}$. Angew. Chem. Int. Ed. 2019, 58, 5038. 
(7) Bačić, Z.; Xu, M.; Felker, P. M. Coupled translation-rotation dynamics of $\mathrm{H}_{2}$ and $\mathrm{H}_{2} \mathrm{O}$ inside $\mathrm{C}_{60}$ : Rigorous quantum treatment. Adv. Chem. Phys. 2018, 163, 195.

(8) Cioslowski, J.; Nanayakkara, A. Endohedral fullerites: A new class of ferroelectric materials. Phys. Rev. Lett. 1992, 69, 2871.

(9) Kohama, Y.; Rachi, T.; Jing, J.; Li, Z.; Tang, J.; Kumashiro, R.; Izumisawa, S.; Kawaji, H.; Atake, T.; Sawa, H. et al. Rotational sublevels of an ortho-hydrogen molecule encapsulated in an isotropic $\mathrm{C}_{60}$ cage. Phys. Rev. Lett. 2009, 103, 073001.

(10) Mamone, S.; Johnson, M. R.; Ollivier, J.; Rols, S.; Levitt, M. H.; Horsewill, A. J. Symmetry-breaking in the $\mathrm{H}_{2} \mathrm{C}_{60}$ endofullerene revealed by inelastic neutron scattering at low temperature. Phys. Chem. Chem. Phys. 2016, 18, 1998.

(11) Beduz, C.; Carravetta, M.; Chen, J. Y. C.; Concistre, M.; Denning, M.; Frunzi, M.; Horsewill, A. J.; Johannessenn, O. G.; Lawler, R.; Lei, X. et al. Quantum rotation of ortho and para-water encapsulated in a fullerene cage. Proc. Natl. Acad. Sci. U.S.A. 2012, 109, 12894.

(12) Goh, K. S. K.; Jiménez-Ruiz, M.; Johnson, M. R.; Rols, S.; Ollivier, J.; Denning, M. S.; Mamone, S.; Levitt, M. H.; Lei, X.; Li, Y. et al. Symmetry-breaking in the endofullerene $\mathrm{H}_{2} \mathrm{O} @ \mathrm{C}_{60}$ revealed in the quantum dynamics of ortho and para-water: a neutron scattering investigation. Phys. Chem. Chem. Phys. 2014, 16, 21330.

(13) A. J. Horsewill has informed us that the observed $1_{01}$ splitting in $\mathrm{H}_{2} \mathrm{O} @ \mathrm{C}_{60}$ is such that the lower energy level is nondegenerate and the upper one is doubly degenerate. Due to a misprint, the opposite ordering was erroneously reported in Goh et al. ${ }^{12}$.

(14) Kalugina, Y. N.; Roy, P. N. Potential energy and dipole moment surfaces for $\mathrm{HF} @ \mathrm{C}_{60}$ : Prediction of spectral and electric response properties. J. Chem. Phys. 2017, 147, 244303. 
(15) Bačić, Z.; Vlček, V.; Neuhauser, D.; Felker, P. M. Effects of symmetry breaking on the translation-rotation eigenstates of $\mathrm{H}_{2}, \mathrm{HF}$, and $\mathrm{H}_{2} \mathrm{O}$ inside the fullerene $\mathrm{C}_{60}$. Faraday Discuss. 2018, 212, 547.

(16) Xu, M.; Sebastianelli, F.; Bačić, Z.; Lawler, R.; Turro, N. J. Quantum dynamics of coupled translational and rotational motions of $\mathrm{H}_{2}$ inside $\mathrm{C}_{60}$. J. Chem. Phys. 2008, $128,011101$.

(17) Xu, M.; Sebastianelli, F.; Bačić, Z.; Lawler, R.; Turro, N. J. $\mathrm{H}_{2}$, HD, and $\mathrm{D}_{2}$ inside $\mathrm{C}_{60}$ : Coupled translation-rotation eigenstates of the endohedral molecules from quantum five-dimensional calculations. J. Chem. Phys. 2008, 129, 064313.

(18) Xu, M.; Sebastianelli, F.; Gibbons, B. R.; Bačić, Z.; Lawler, R.; Turro, N. J. Coupled translation-rotation eigenstates of $\mathrm{H}_{2}$ in $\mathrm{C}_{60}$ and $\mathrm{C}_{70}$ on the spectroscopically optimized interaction potential: Effects of cage anisotropy on the energy level structure and assignments. J. Chem. Phys. 2009, 130, 224306.

(19) Mamone, S.; Ge, M.; Hüvonen, D.; Nagel, U.; Danquigny, A.; Cuda, F.; Grossel, M. C.; Murata, Y.; Komatsu, K.; Levitt, M. H. et al. Rotor in a cage: Infrared spectroscopy of an endohedral hydrogen-fullerene complex. J. Chem. Phys. 2009, 130, 081103.

(20) Felker, P. M.; Vlček, V.; Hietanen, I.; FitzGerald, S.; Neuhauser, D.; Bačić, Z. Explaining the symmetry breaking observed in the endofullerenes $\mathrm{H}_{2} @ \mathrm{C}_{60}, \mathrm{HF}_{\mathrm{O}} \mathrm{C}_{60}$, and $\mathrm{H}_{2} \mathrm{O} @ \mathrm{C}_{60}$. Phys. Chem. Chem. Phys. 2017, 19, 31274.

(21) Xu, M.; Ulivi, L.; Celli, M.; Colognesi, D.; Bačić, Z. Quantum calculation of inelastic neutron-scattering spectra of a hydrogen molecule inside a nanoscale cavity based on rigorous treatment of the coupled translation-rotation dynamics. Phys. Rev. B 2011, 83, 241403(R).

(22) Xu, M.; Bačić, Z. Inelastic neutron scattering spectra of a hydrogen molecule in a 
nanocavity: Methodology for quantum calculations incorporating the coupled fivedimensional translation-rotation eigenstates. Phys. Rev. B 2011, 84, 195445.

(23) Xu, M.; Ulivi, L.; Celli, M.; Colognesi, D.; Bačić, Z. Rigorous quantum treatment of inelastic neutron scattering spectra of a heteronuclear diatomic molecule in a nanocavity: HD in the small cage of structure II clathrate hydrate. Chem. Phys. Lett. 2013, $563,1$.

(24) Xu, M.; Ye, S.; Powers, A.; Lawler, R.; Turro, N. J.; Bačić, Z. Inelastic neutron scattering spectrum of $\mathrm{H}_{2} @ \mathrm{C}_{60}$ and its temperature dependence decoded using rigorous quantum calculations and a new selection rule. J. Chem. Phys. 2013, 139, 064309.

(25) Xu, M.; Jiménez-Ruiz, M.; Johnson, M. R.; Rols, S.; Ye, S.; Carravetta, M.; Denning, M. S.; Lei, X.; Bačić, Z.; Horsewill, A. J. Confirming a predicted selection rule in inelastic neutron scattering spectroscopy: The quantum translator-rotator $\mathrm{H}_{2}$ entrapped inside $\mathrm{C}_{60}$. Phys. Rev. Lett. 2014, 113, 123001.

(26) Xu, M.; Ye, S.; Bačić, Z. General selection rule in the inelastic neutron scattering spectroscopy of a diatomic molecule confined inside a near-spherical nanocavity. J. Phys. Chem. Lett. 2015, 6, 3721.

(27) Ramirez-Cuesta, A. J.; Jones, M. J.; David, W. I. F. Neutron scattering and hydrogen storage. Materials Today 2009, 12, (11) 54.

(28) Kearley, G. J.; Johnson, M. R. Vibrational spectroscopy with neutrons - Where are we now? Vib. Spectrosc. 2010, 53, 54.

(29) Hudson, B. S. Vibrational spectroscopy using inelastic neutron scattering: Overview and outlook. Vib. Spectrosc. 2006, 42, 25.

(30) Mitchell, P. C. H.; Parker, S. F.; Ramirez-Cuesta, A. J.; Tomkinson, J. Vibrational Spectroscopy with Neutrons; World Scientific: Singapore, 2005. 
(31) Poirier, B. Communication: The $\mathrm{H}_{2} @ \mathrm{C}_{60}$ inelastic neutron scattering selection rule: Expanded and explained. J. Chem. Phys. 2015, 143, 101104.

(32) Xu, M.; Ye, S.; Lawler, R.; Turro, N. J.; Bačić, Z. HD in $\mathrm{C}_{60}$ : theoretical prediction of the inelastic neutron scattering spectrum and its temperature dependence. Phil. Trans. R. Soc. A 2013, 371, 20110630. 
Table 1: Intensities, in barn (bn), of the INS transitions from the ground TR state of $\mathbf{H F} @ \mathbf{C}_{60}$, calculated for the isolated endofullerene $\left(I_{0}\right)$ and for the $\mathbf{P}$ crystal orientation $\left(I_{P}\right)$, respectively; incident neutron wavelength $\lambda_{\mathrm{n}}=1.65 \AA$. $\Delta E_{0}$ and $\Delta E_{P}$ (in meV) are the neutron energy transfer (level energy relative to the TR ground state) for the isolated $\mathbf{H F} @ \mathbf{C}_{60}$ and the $\mathbf{P}$ orientation, respectively. $g_{0}$ and $g_{P}$ are the respective degeneracies of these TR levels, while $(n, l, j), \lambda$, and $\left|m_{\lambda}\right|$ are their quantum numbers. The bold-faced transitions are those that for isolated $\mathrm{HF} @ \mathrm{C}_{60}$ are forbidden according to the selection rule stated in the text.

\begin{tabular}{|c|c|c|c|c|c|c|c|c|c|}
\hline$\Delta E_{0}$ & $g_{0}$ & $(n, l, j)$ & $\lambda$ & $I_{0}(\mathrm{bn})$ & $\Delta E_{P}$ & $g_{P}$ & $\lambda$ & $\left|m_{\lambda}\right|$ & $I_{P}(\mathrm{bn})$ \\
\hline 0.00 & 1 & $(0,0,0)$ & 0 & 10.9367 & 0.00 & 1 & 0 & 0 & 10.9411 \\
\hline \multirow[t]{2}{*}{4.178} & 3 & $(0,0,1)$ & 1 & 8.0673 & 3.876 & 1 & 1 & 0 & 2.8716 \\
\hline & & & & & 4.337 & 2 & 1 & 1 & 5.1935 \\
\hline \multirow[t]{2}{*}{9.466} & 3 & $(1,1,0)$ & 1 & 1.5909 & 9.452 & 1 & 1 & 0 & 0.5021 \\
\hline & & & & & 9.475 & 2 & 1 & 1 & 1.0933 \\
\hline \multirow[t]{3}{*}{11.253} & 5 & $(1,1,1)$ & 2 & 1.8199 & 11.056 & 1 & 2 & 0 & 0.3897 \\
\hline & & & & & 11.150 & 2 & 2 & 1 & 0.7410 \\
\hline & & & & & 11.454 & 2 & 2 & 2 & 0.6695 \\
\hline 13.407 & 1 & $(1,1,1)$ & 0 & 0.2356 & 13.412 & 1 & 0 & 0 & 0.2465 \\
\hline \multirow[t]{2}{*}{13.514} & 3 & $(\mathbf{1}, \mathbf{1}, \mathbf{1})$ & 1 & 0.0000 & 13.440 & 2 & 1 & 1 & 0.0450 \\
\hline & & & & & 13.683 & 1 & 1 & 0 & 0.0000 \\
\hline \multirow[t]{3}{*}{15.319} & 5 & $(0,0,2)$ & 2 & 5.0708 & 15.167 & 1 & 2 & 0 & 1.0311 \\
\hline & & & & & 15.251 & 2 & 2 & 1 & 2.0252 \\
\hline & & & & & 15.500 & 2 & 2 & 2 & 1.9709 \\
\hline$\ldots$ & & $\cdots$ & &. & . & & & & \\
\hline \multirow[t]{3}{*}{21.205} & 5 & $(2,2,1)$ & 2 & 0.0000 & 21.207 & 2 & 2 & 2 & 0.0004 \\
\hline & & & & & 21.222 & 1 & 2 & 0 & 0.0000 \\
\hline & & & & & 21.264 & 2 & 2 & 1 & 0.0240 \\
\hline$\cdots$ & & $\cdots$ & & . & $\cdots$ & & & & $\cdots$ \\
\hline \multirow[t]{3}{*}{24.115} & 5 & $(1,1,2)$ & 2 & 0.0000 & 24.157 & 2 & 2 & 1 & 0.0093 \\
\hline & & & & & 24.162 & 1 & 2 & 0 & 0.0000 \\
\hline & & & & & 24.170 & 2 & 2 & 2 & 0.0145 \\
\hline
\end{tabular}


a)

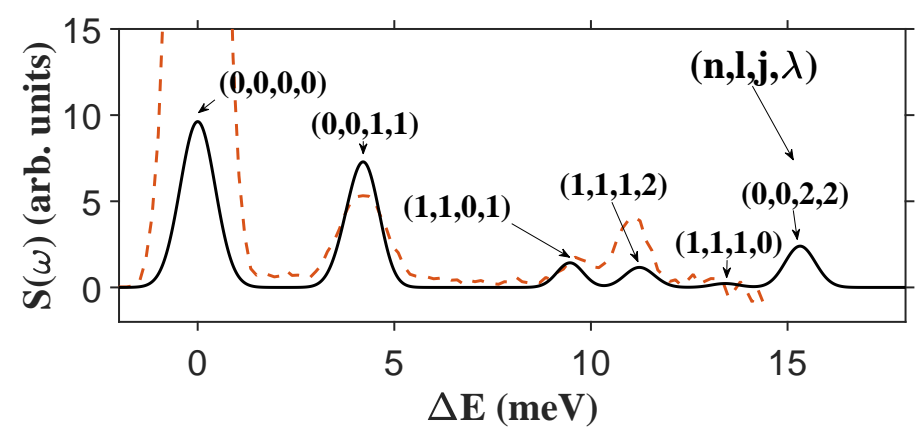

b)

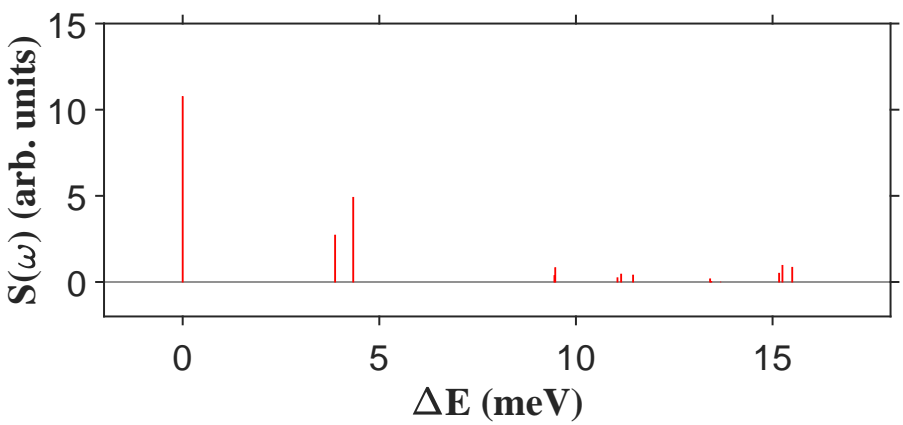

c)

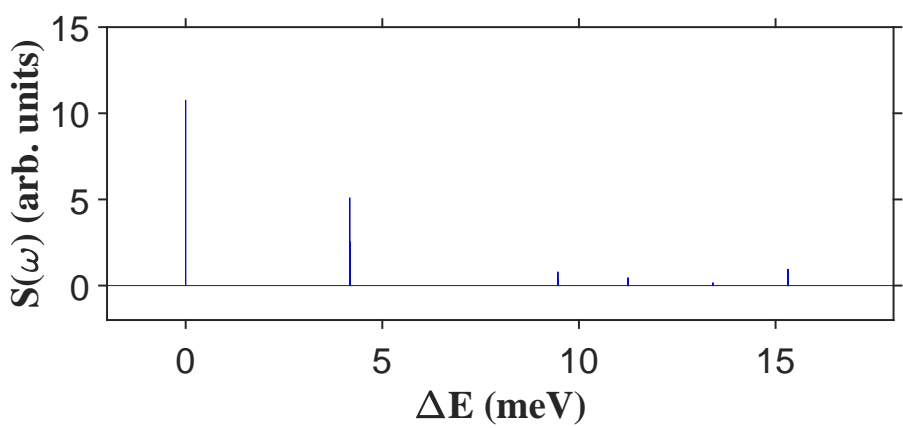

Figure 1: INS spectra of $\mathrm{HF} @ \mathrm{C}_{60}(s)$ computed for $0 \mathrm{~K}$ and the incident neutron wavelength $\lambda_{\mathrm{n}}=2.22 \AA$. All transitions originate from the ground TR state of HF. (a) The spectrum computed for a $15 \%$ contribution from the $\mathrm{H}$ orientation and $85 \%$ from the $\mathrm{P}$ orientation. The resulting stick spectrum convolved with the instrumental resolution function is depicted with the solid black line. The calculated transitions are labeled with the quantum numbers $(n, l, j, \lambda)$ of their final TR states. Dashed red line shows the experimental INS spectrum ${ }^{5}$ recorded at $1.6 \mathrm{~K}$ and for $\lambda_{\mathrm{n}}=2.22 \AA$. (b) The computed spectrum for the $\mathrm{P}$ orientation. (c) The computed spectrum for the $\mathrm{H}$ orientation. 


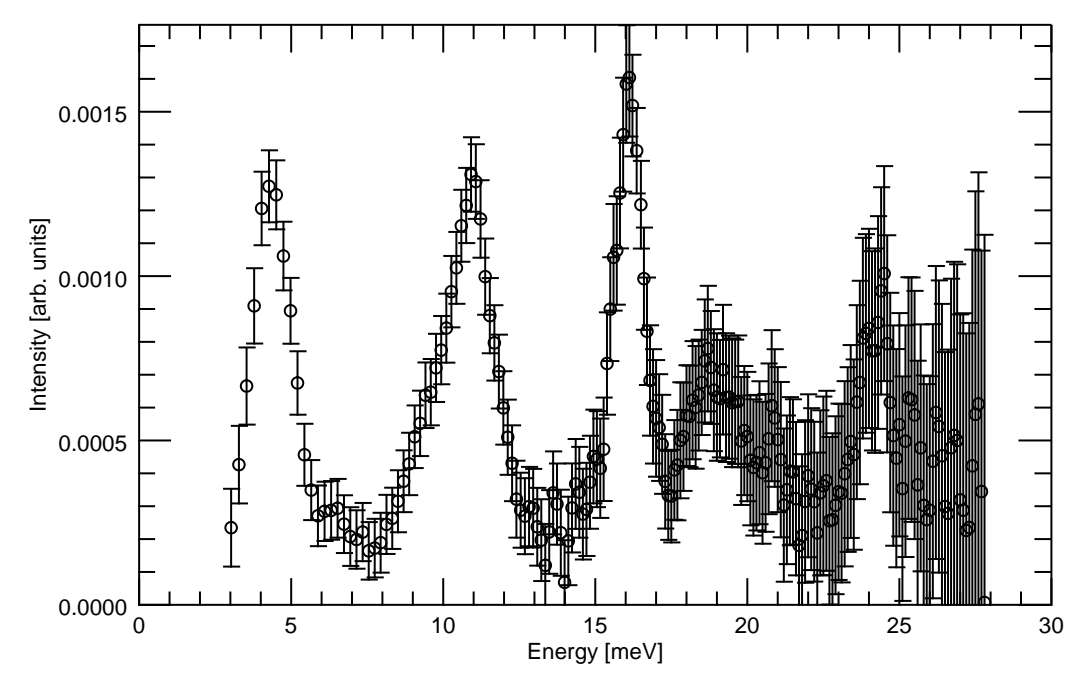

Figure 2: The INS spectrum of $\mathrm{HF}_{6} \mathrm{C}_{60}$ recorded at $1.5 \mathrm{~K}$, using the incident neutron wavelength $\lambda_{\mathrm{n}}=1.60 \AA$. 
a)

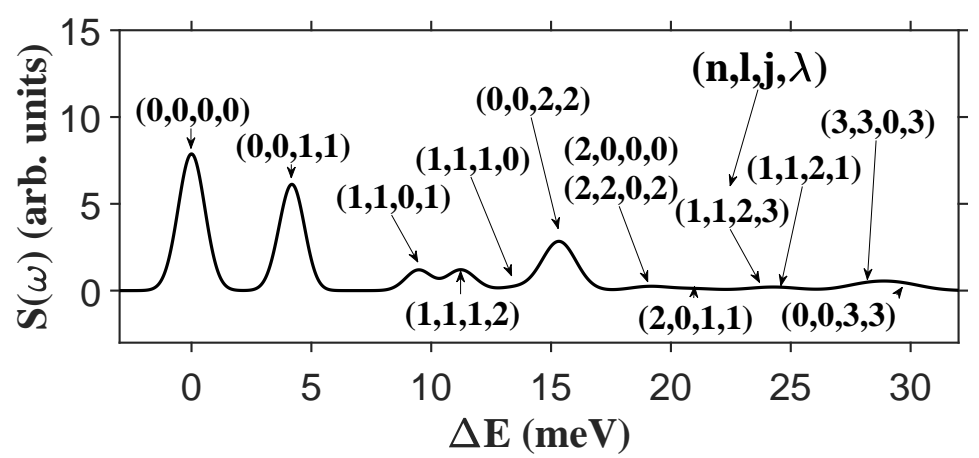

b)

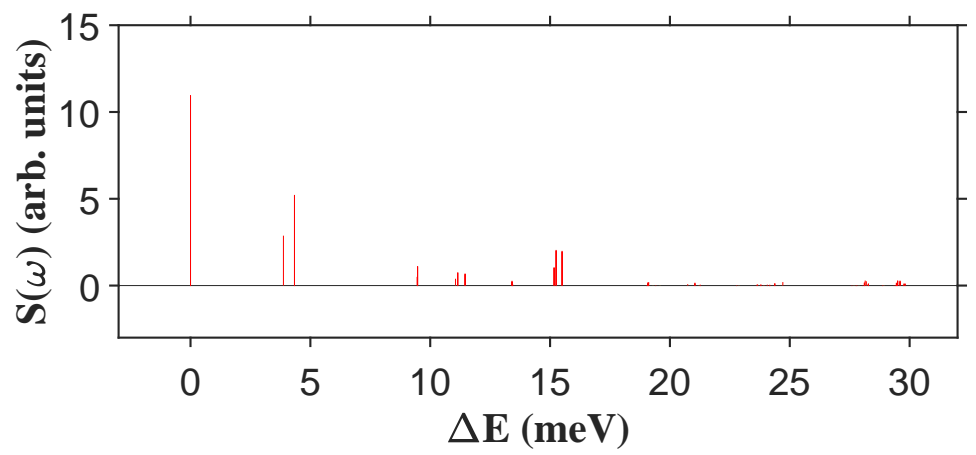

c)

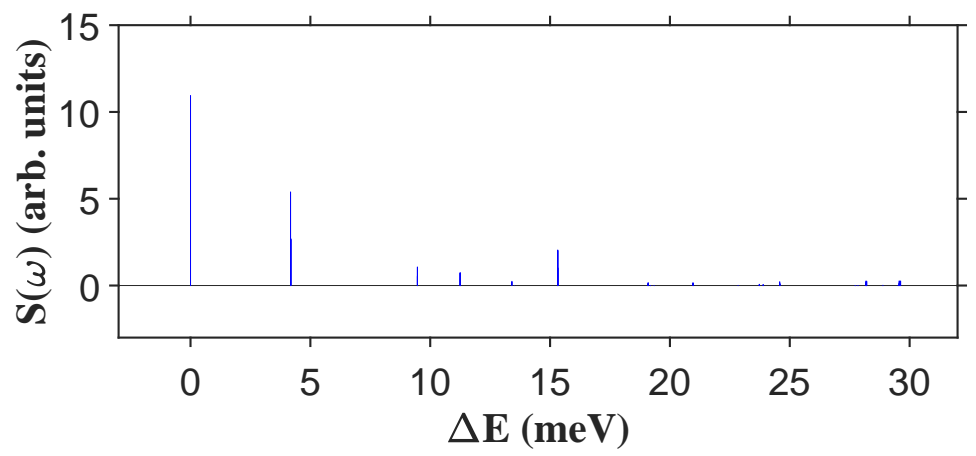

Figure 3: Calculated INS spectra of $\mathrm{HF}_{6} @ \mathrm{C}_{60}(s)$, for $0 \mathrm{~K}$ and the incident neutron wavelength $\lambda_{\mathrm{n}}=1.65 \AA$. All transitions originate from the ground TR state of HF. (a) The spectrum computed for a $15 \%$ contribution from the $\mathrm{H}$ orientation and $85 \%$ from the $\mathrm{P}$ orientation. The resulting stick spectrum convolved with the instrumental resolution function is depicted with the solid black line. (b) The spectrum for the $\mathrm{P}$ orientation. (c) The spectrum for the $\mathrm{H}$ orientation. 